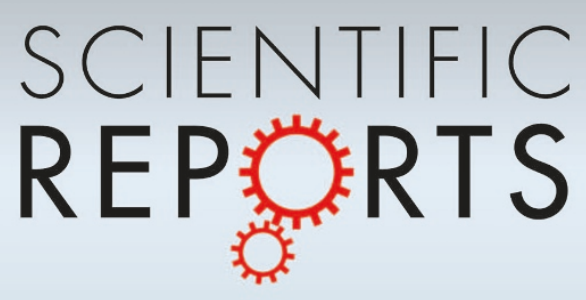

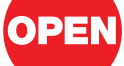

SUBJECT AREAS:

PHOTOCATALYSIS

TWO-DIMENSIONAL MATERIALS

SURFACES, INTERFACES AND

THIN FILMS

DENSITY FUNCTIONAL THEORY

Received

12 March 2013

Accepted

29 April 2013

Published

17 May 2013

Correspondence and requests for materials should be addressed to J.Y. (jlyang@ustc.edu.

$\mathrm{cn})$

\section{Semihydrogenated BN Sheet: A Promising Visible-light Driven Photocatalyst for Water Splitting}

\author{
Xingxing Li, Jin Zhao \& Jinlong Yang
}

Hefei National Laboratory of Physical Science at the Microscale, University of Science and Technology of China, Hefei, Anhui 230026, China.

Based on first principles calculations, we predict semihydrogenated graphitic BN (sh-BN) sheet is a potential metal-free visible-light driven photocatalyst for water splitting. The ground state of sh-BN is a strip-like antiferromagnetic semiconductor with a band gap suitable for visible-light absorption. The redox potentials of water splitting are all located inside the band gap and the probability densities of valence and conduction bands are distributed apart spatially leading to a well-separation of photogenerated electrons and holes.

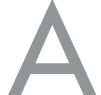

$s$ an ultimate clean energy, hydrogen produced through photocatalytic water decomposition using solar energy plays an important role in solving energy and environmental problems. The key of solar hydrogen production is to develop photocatalysts active under visible light. Up to now, most of photocatalysts are discovered in metal oxides, sulfides and nitrides with $d^{0}$ or $d^{10}$ transition metal cations ${ }^{1-8}$, for example, $\mathrm{TiO}_{2}$, is among the most promising ones. Unfortunately, most of these catalysts are active only under UV irradiation, while others absorbing visible light are not stable during the reaction process, such as photocorrosion in CdS?. Photocatalysts for practical use with relatively high productivity are not found yet. Searching for new efficient photocatalysts both from theoretical and experimental aspects is urgently needed.

Metal-free photocatalysts may have the advantage of non-toxicity and good processability, and are gradually becoming an important catalyst developing. The first organic semiconductor used for photoreduction of water to hydrogen is poly(p-phenylene) though it is only active under ultraviolet light ${ }^{10}$. Recent discovered graphitic carbon nitride, with a band gap of $2.7 \mathrm{eV}$ to absorb blue light, is a good candidate photocatalyst for water splitting ${ }^{11,12}$. This is the first report on generating hydrogen from water under visible light in the absence of transition metals. It opens up a new way to searching for more metal-free photocatalysts. Based on first principles calculations, we here report semihydrogenated BN sheet (sh-BN) as another candidate of visible-light driven photocatalysts that is simple and reachable, containing no transition metals. After carefully examining the magnetic coupling induced in the structure, we find a strip-like antiferromagnetic ground state. The semiconducting sh-BN sheet has a band gap around $2.24 \mathrm{eV}$, which is suitable for visible-light adsorption. The redox potentials of $\mathrm{H}_{2} \mathrm{O}$ are both located in between the band gap which indicates that it is a good candidate for water splitting.

\section{Results}

Firstly we have investigated the ground state of the sh-BN sheet. For graphitic BN sheet, two ways of semihydrogenation are considered: on boron sublattice and on nitrogen sublattice. Our results show that semihydrogenation on boron sublattice is energetically preferred than on nitrogen sublattice by $0.35 \mathrm{eV}$ per primitive cell. Therefore we mainly focus on the first configuration. From the optimized structure in Figure 1, one can see that hydrogenation on boron sublattice changes the graphitic BN sheet from a planar structure to a buckled one with the distance of $0.27 \AA$ between the boron plane and nitrogen plane. We then examine the thermodynamic stability of sh-BN, and calculate the desorption energy of hydrogen to be $0.31 \mathrm{eV}$ per $\mathrm{H}$ atom, indicating the desorbing is endothermic and unlikely. Moreover, as the sh-BN with hydrogenation on $\mathrm{B}$ sublattice is $0.35 \mathrm{eV}$ per $\mathrm{H}$ atom lower in energy than that on $\mathrm{N}$ sublattice, the diffusion barrier of $\mathrm{H}$ atom from $\mathrm{B}$ atom to neighboring $\mathrm{N}$ atom would be at least $0.35 \mathrm{eV}$. So it is not easy for the $\mathrm{H}$ atoms to diffuse together and couple each other. This ensures the good stability of sh-BN. Due to the spin polarization of the unpaired $p_{z}$ electrons on unhydrogenated nitrogen atoms, each primitive cell carries a magnetic moment of $1 \mu_{\mathrm{B}}$ among which nitrogen atom carries the most part 


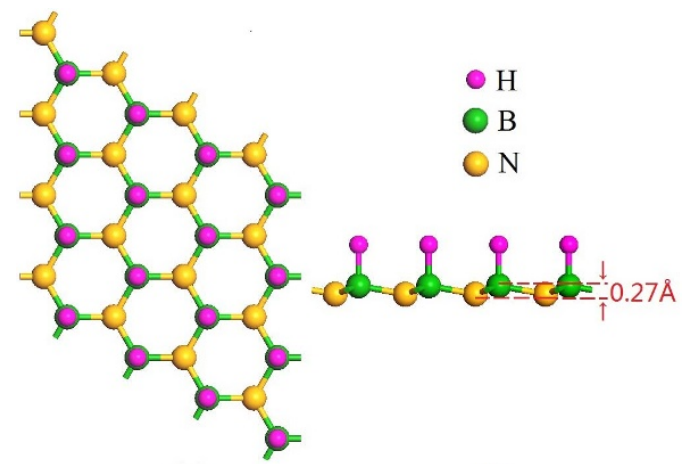

(a)

(b)
Figure 1 The optimized structure of sh-BN under (a) top view and (b) side view.

and hydrogen atom possesses the small part. In order to find the magnetic ground state, three magnetic configurations in Figure 2 are considered. The first two configurations are what we usually referred to as ferromagnetic (FM) and antiferromagnetic (AFM) coupling in analogous systems. The FM state is more stable than AFM state as also shown by previous work ${ }^{13}$. However, the FM state is not the ground state, and it is $0.32 \mathrm{eV}$ higher in energy than the strip-like AFM state in Figure $2 \mathrm{c}$ where the spin density in the same direction forms strips arranged alternately. The nonmagnetic (NM) state is also calculated, which is much less stable than strip-like AFM state with an energy difference of about $0.97 \mathrm{eV}$ per supercell.

Due to the size of supercell, each strip formed in the strip-like AFM state in Figure 2c contains only two lines of nitrogen (boron, hydrogen) atoms so that we can refer to the magnetic coupling as 'two lines spin up, two lines spin down' represented by $2 \uparrow 2 \downarrow$. We then systematically studied the relative stability of strip-like AFM state $\mathrm{N} \uparrow \mathrm{N} \downarrow$ with $\mathrm{N}=1,2, \ldots, 8$ using a rectangular supercell illustrated in Figure 3a. Actually, $\mathrm{N}=1$ corresponds to the AFM state in Figure $2 \mathrm{~b}$. The results in Figure $3 \mathrm{~b}$ show that when $\mathrm{N}=1, \mathrm{E}(1 \uparrow 1 \downarrow)>\mathrm{E}(\mathrm{FM})$; while $\mathrm{N}>1, \mathrm{E}(\mathrm{N} \uparrow \mathrm{N} \downarrow)<\mathrm{E}(\mathrm{FM})$. The minimum point occurs at $\mathrm{N}=$ 2 which indicates the $2 \uparrow 2 \downarrow$ state is the most stable, i.e. ground state of sh-BN.

The activity of photocatalysts is related significantly to the band gap and the position of conduction band (CB) and valance band (VB) of the material. Bare graphitic $\mathrm{BN}$ sheet is known to be an insulator with a band gap of $4.64 \mathrm{eV}^{14}$ which is largely reduced after semihydrogenation as shown in Figure 4a. However, it is known that PBE functional usually underestimates the band gap. In order to get

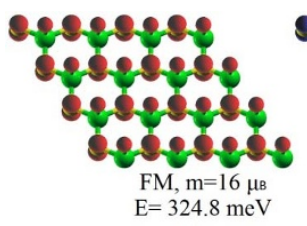

(a)

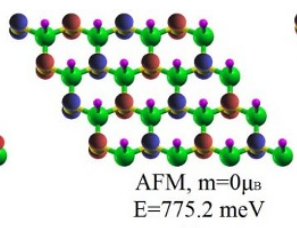

(b)

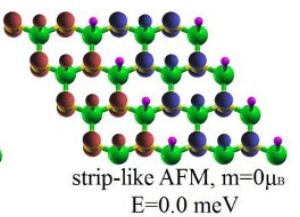

(c)
Figure $2 \mid$ The spin density of sh-BN under three different magnetic coupling with an isovalue of $0.22 \mathrm{e} / \AA^{3}$. Red and blue indicate the positive (spin up) and negative (spin down) values, respectively. Green, yellow and purple balls represent boron, nitrogen and hydrogen atoms, respectively.

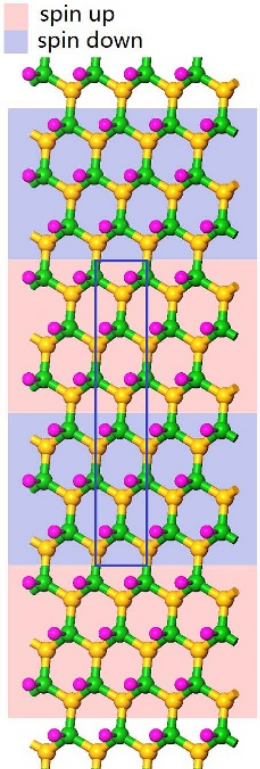

(a)

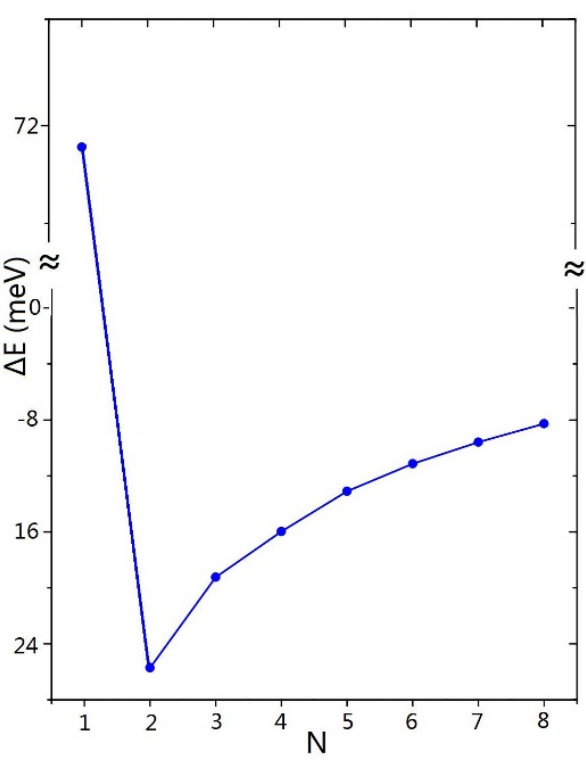

(b)
Figure $3 \mid$ (a) Illustration of strip-like AFM state: $3 \uparrow 3 \downarrow$. (b) The relative stability of $\mathrm{N} \uparrow \mathrm{N} \downarrow$ state where $\Delta E(m e V)=[E(N \uparrow N \downarrow)-E(F M)] / N$.

accurate electronic structure, we then employ the screened hybrid functional HSE06 ${ }^{15,16}$, which can provide band gaps in good agreement with experiment for small- to medium-gap systems ${ }^{17,18}$. To evaluate the accuracy of our HSE06 procedure, the band gap of pure $\mathrm{BN}$ sheet is computed to be $5.69 \mathrm{eV}$, very close to the experiment value of $5.56 \mathrm{eV}^{19}$. As with the sh-BN, the band gap got by HSE06 is
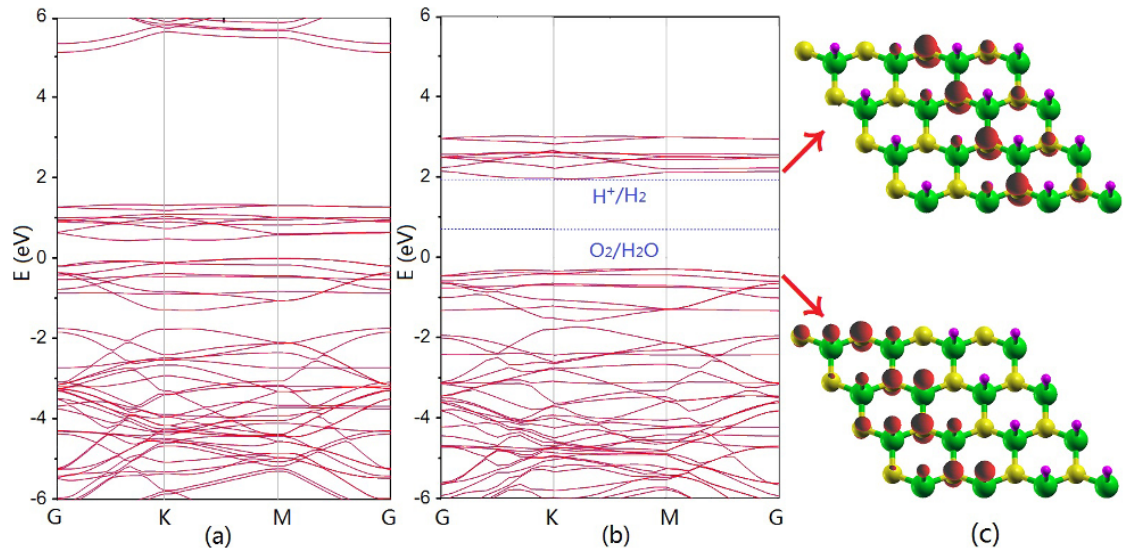

Figure 4| The band structure of sh-BN with (a) PBE functional and (b) HSE06 functional. The position of the reduction level for $\mathrm{H}^{+}$to $\mathrm{H}_{2}$ and the oxidation potential of $\mathrm{H}_{2} \mathrm{O}$ to $\mathrm{O}_{2}$ is indicated by the blue dashed line. The Fermi Level is set to zero. (c) The charge distribution of VB (the lower part) and $\mathrm{CB}$ (the upper part) with isovalue $0.03 \mathrm{e} / \AA^{3}$. 


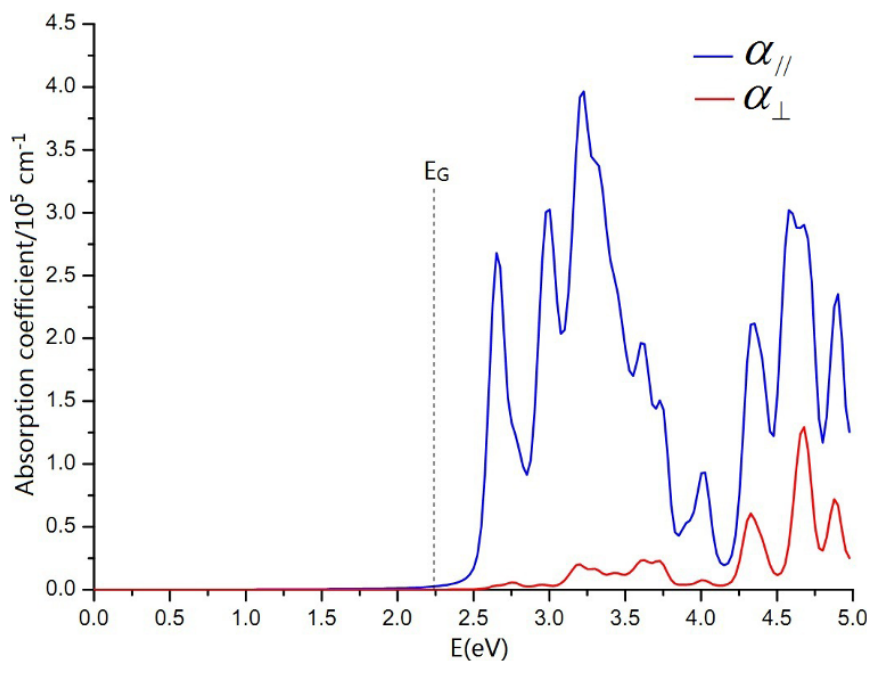

Figure $5 \mid$ The calculated optical absorption for sh-BN with the polarization vector parallel $\left(\alpha_{/ /}\right)$and perpendicular $\left(\alpha_{\perp}\right)$ to z-axis.

$2.24 \mathrm{eV}$ corresponding to an absorption in the yellow-green region of the visible spectrum. For use in photocatalytic water splitting, the band gap of the photocatalyst must be sufficiently large to overcome the endothermic character of the water-splitting reaction, i.e. larger than $1.23 \mathrm{eV}^{11}$; moreover, to be visible-light active, it should be narrower than $3.0 \mathrm{eV}$. These two conditions are obviously satisfied for the sh-BN sheet. Nevertheless, for higher sufficiency: both the reduction potential $\left(V_{\mathrm{H}^{+} / \mathrm{H}_{2}}=4.44 \mathrm{eV}\right)$ for $\mathrm{H}^{+}$to $\mathrm{H}_{2}$ and the oxidation potential $\left(V_{\mathrm{O}_{2} / \mathrm{H}_{2} \mathrm{O}}=5.67 \mathrm{eV}\right)$ of $\mathrm{H}_{2} \mathrm{O}$ to $\mathrm{O}_{2}$ should be located inside the band gap ${ }^{20}$. From Figure $4 \mathrm{~b}$, for the sh-BN sheet, it is clear that the reduction level is slightly below the $\mathrm{CB}$, while the oxidation level is in between the gap. This reveals the oxidation process is energetically favored and the reduction is permitted presumably with a relatively low driving force. Briefly, from thermodynamic aspect, the sh-BN is a good candidate as photocatalyst for water splitting.

Another important point for photocatalysis is the good separation of photogenerated electron-hole pairs. Recombination of photogenerated electrons and holes can cause a big decrease in the photocatalytic activity. In Figures $4 \mathrm{c}$, one can see that the probability distribution of $\mathrm{VB}$ and $\mathrm{CB}$ is well separated spatially: the former is localized in the left part of the supercell whereas the latter is mainly in the right part, leading to a low probability of recombination. This ascertains the sh-BN as a potential photocatalyst for water splitting. Moreover, in order to see whether the charge separation can influence the absorption of light, we have calculated the optical absorption spectrum of sh-BN with HSE06 functional. Firstly, the frequency-dependent dielectric function $\varepsilon(\omega)=\varepsilon_{1}(\omega)+i \varepsilon_{2}(\omega)$ is calculated, and then the absorption coefficient as a function of photon energy is evaluated according to the following expression:

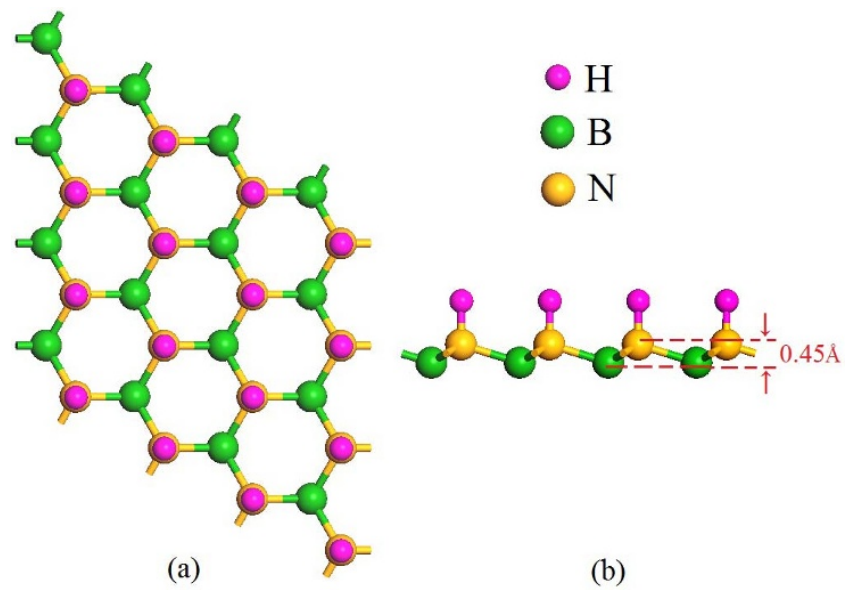

Figure 6 | The optimized structure of semihydrogenation on nitrogen sublattice of graphitic BN sheet under (a) top view and (b) side view.

$$
\alpha(E)=\frac{4 \pi e}{h c}\left\{\frac{\left[\varepsilon_{1}^{2}+\varepsilon_{2}^{2}\right]^{1 / 2}-\varepsilon_{1}}{2}\right\}^{1 / 2}
$$

The results are shown in Figure 5. We can see that as a result of structural anisotropy, different absorption behaviors occur in the transversal and longitudinal direction: the absorption coefficient in the former direction is much larger than in the latter one. And the sh$\mathrm{BN}$ shows a strong absorption in the visible-light region, with the intensity up to $10^{5} \mathrm{~cm}^{-1}$, which dispels our worry about notable decrease in the absorption intensity due to the charge separation and ensures the sh-BN is visible-light active.

The sh-BN with hydrogenation on nitrogen sublattice is also investigated. Figure 6 gives a buckle height of $0.45 \AA$. The produced structure also prefers a strip-like AFM state, which is nearly degenerate with the usually said AFM state (Figure 7). And the electronic band structure under HSE06 functional indicates a semiconductor with a band gap of $1.50 \mathrm{eV}$ (Figure 8). Though the oxidation level of $\mathrm{H}_{2} \mathrm{O}$ to $\mathrm{O}_{2}$ is outside the band gap, the reduction level for $\mathrm{H}^{+}$to $\mathrm{H}_{2}$ is still located in the gap which indicates the material may be a good photocatalyst for $\mathrm{H}_{2}$ evolution under visible light if a hole scavenger exists 9 .

\section{Discussion}

Our findings have broad implications for designing new metal-free photocatalysts. Atomic thick two dimensional nanosheets, for example, honeycomb monolayer of group-IV elements and III-V binary compounds such as graphene ${ }^{21}$, hexagonal $\mathrm{SiC}$ and $\mathrm{BN}$ sheet ${ }^{22,23}$, and recently synthesized silicene $e^{24}$, are all possible candidate precursors. The means of chemical functionalization here is not limited to

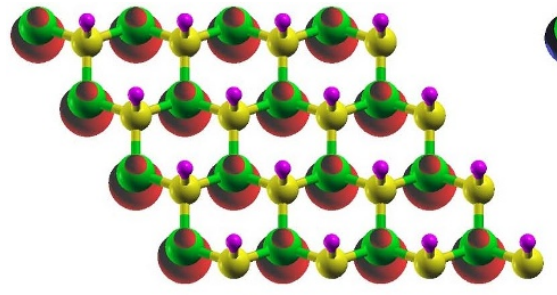

$\mathrm{FM}, \mathrm{m}=11.9 \mu_{\mathrm{B}}$

$\mathrm{E}=1186.2 \mathrm{meV}$

(a)

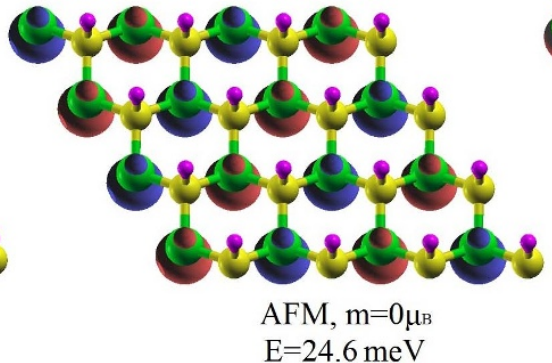

(b)

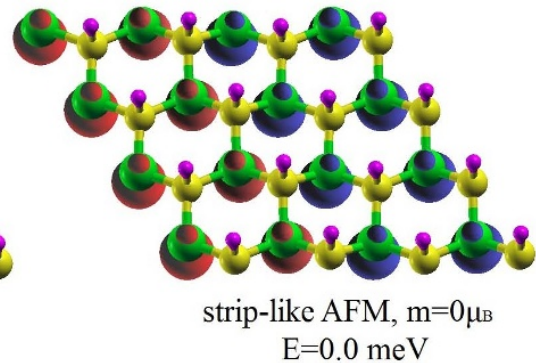

(c)

Figure $7 \mid$ The relative stability of three different magnetic coupling in the case of semihydrogenation on nitrogen sublattice. Spin densities are plotted with an isovalue of $0.12 \mathrm{e} / \AA^{3}$. Red and blue indicate spin up and spin down, respectively. 


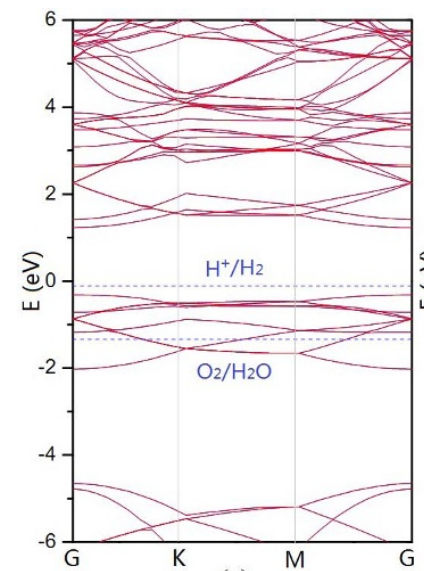

(a)

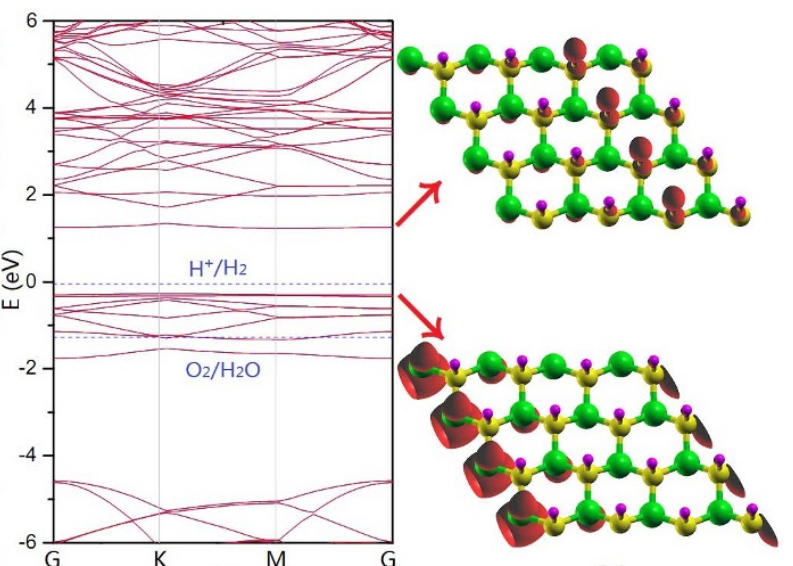

(b) (c)

Figure 8 $\mid$ The electronic band structure for graphitic BN sheet semihydrogenated on nitrogen sublattice under (a) AFM state and (b) strip-like AFM state with HSE06 functional. The Fermi Level is set to zero. The redox potentials of water splitting are shown by blue dashed lines. (c) The charge density of valence band (the lower part) and conduction band (the upper part) under strip-like AFM state with isovalue $0.01 \mathrm{e} / \AA^{3}$.

hydrogenation; many other methods like fluorination, hydroxylation and amination can also be feasible to reach our goals $\mathrm{s}^{25-28}$.

In conclusion, we have discovered the sh-BN to be a new potential two-dimensional metal-free photocatalyst for water splitting under visible light. After semihydrogenation, the magnetic and electronic properties of graphitic BN sheet are largely changed. It prefers a striplike antiferromagnetic coupling with no net magnetization. Then from our analysis of electronic structure using screened hybrid functional, the idea of using the sh-BN sheet as a photocatalyst for water splitting is verified. We expect that the facility of chemical functionalizing analogous two dimensional materials will lead to the discovery of new promising metal-free photocatalysts.

\section{Methods}

All our calculations are carried out within the Perdew-Burke-Ernzerholf generalized gradient approximation ${ }^{29}$ implemented in the Vienna ab Initio Simulation Package ${ }^{30}$. Projector augmented wave (PAW) potentials and a plane-wave cut-off energy of $400 \mathrm{eV}$ are used ${ }^{31}$. The vacuum space is set to be $15 \AA$ in order to avoid interactions between two nearest-neighbor sheets. Due to the long-range magnetic interaction exist in analogous metal-free magnetic systems ${ }^{32}$, the $2 \times 2$ supercell one usually uses may not be sufficient in finding ground magnetic state. So we employ a supercell consisting of four primitive cells in each lattice direction to study the electronic structure. The first Brillouin zone is sampled with Monkhorst-Pack grid of $5 \times 5 \times 1$ for structure optimization and $9 \times 9 \times 1$ for static calculation. The criterion of convergence for energy and force are set to be $10^{-6} \mathrm{eV}$ and $0.01 \mathrm{eV} / \AA$, respectively.

As for the calculation of frequency-dependent dielectric function, we first calculate its imaginary part by a summation over empty states using the equation ${ }^{33}$ :

$\varepsilon_{\alpha \beta}^{(2)}(\omega)=\frac{4 \pi^{2} e^{2}}{\Omega} \lim _{q \rightarrow 0} \frac{1}{q^{2}} \sum_{c, v, \mathbf{k}} 2 \omega_{\mathbf{k}} \delta\left(E_{c \mathbf{k}}-E_{v \mathbf{k}}-\omega\right) \times\left\langle\mu_{c \mathbf{k}+\mathbf{e}_{x} \mathbf{q}} \mid \mu_{v \mathbf{k}}\right\rangle\left\langle\mu_{c \mathbf{k}+\mathbf{e}_{\beta} \mathbf{q}} \mid \mu_{v \mathbf{k}}\right\rangle^{*}$

where the indices $c$ and $v$ refer to conduction and valence band states respectively, and $\mu_{\mathrm{ck}}$ is the cell periodic part of the wavefunctions at the k-point $\mathbf{k}$. Then the real part of the dielectric tensor $\varepsilon_{\alpha \beta}^{(2)}(\omega)$ is derived from $\varepsilon_{\alpha \beta}^{(2)}(\omega)$ by the usual Kramers-Kronig relationship.

1. Yamaguti, K. \& Sato, S. Photolysis of Water over Metallized Powdered Titanium Dioxide. J. Chem. Soc., Faraday Trans. 1 81, 1237-1246 (1985).

2. Domen, K., Naito, S., Soma, M., Onishi, T. \& Tamaru, K. Photocatalytic Decomposition of Water Vapour on a $\mathrm{NiO}_{-} \mathrm{SrTiO}_{3}$ Catalyst. J. Chem. Soc., Chem. Commun. 543-544 (1980).

3. Sato, J., Kobayashi, H., Saito, N., Nishiyama, H. \& Inoue, Y. Photocatalytic Activities for Water Decomposition of $\mathrm{RuO}_{2}$-loaded $\mathrm{AInO}_{2}(\mathrm{~A}=\mathrm{Li}, \mathrm{Na})$ with $\mathrm{d}^{10}$ Configuration. J. Photochem. Photobiol., A 158, 139-144 (2003).

4. Sakata, Y., Matsuda, Y., Yanagida, T., Hirata, K., Imamura, H. \& Teramura, K. Effect of Metal Ion Addition in a Ni Supported $\mathrm{Ga}_{2} \mathrm{O}_{3}$ Photocatalyst on the Photocatalytic Overall Splitting of $\mathrm{H}_{2} \mathrm{O}$. Catal. Lett. 125, 22-26 (2008).

5. Sato, J. et al. $\mathrm{RuO} 2$-Loaded $\beta-\mathrm{Ge}_{3} \mathrm{~N}_{4}$ as a Non-Oxide Photocatalyst for Overall Water Splitting. J. Am. Chem. Soc. 127, 4150-4151 (2005).
6. Maeda, K., Teramura, K., Saito, N., Inoue, Y. \& Domen, K. Photocatalytic Overall Water Splitting on Gallium Nitride Powder. Bull. Chem. Soc. Jpn. 80, 1004-1010 (2007).

7. Matsumura, M., Saho, Y. \& Tsubomura, H. Photocatalytic Hydrogen Production from Solutions of Sulfite using Platinized Cadmium Sulfide Powder. J. Phys. Chem. 87, 3807-3808 (1983).

8. Reber, J. F. \& Meier, K. Photochemical Production of Hydrogen with Zinc Sulfide Suspensions. J. Phys. Chem. 88, 5903-5913 (1984).

9. Kudo, A. \& Miseki, Y. Heterogeneous Photocatalyst Materials for Water Splitting. Chem. Soc. Rev. 38, 253-278 (2009).

10. Yanagida, S., Kabumoto, A., Mizumoto, K., Pac, C. \& Yoshino, K. Poly(para)phenylene-catalyzed Photoreduction of Water to Hydrogen. J. Chem. Soc., Chem. Commun. 8, 474-475 (1985).

11. Wang, X. C. et al. A Metal-free Polymeric Photocatalyst for Hydrogen Production from Water under Visible Light. Nat. Mater. 8, 76-80 (2009).

12. Wang, Y., Wang, X. C. \& Antonietti, M. Polymeric Graphitic Carbon Nitride as a Heterogeneous Organocatalyst: From Photochemistry to Multipurpose Catalysis to Sustainable Chemistry. Angew. Chem. Int. Ed. 51, 68-89 (2012).

13. Zhou, J., Wang, Q., Sun, Q. \& Jena, P. Electronic and Magnetic Properties of a BN Sheet Decorated with Hydrogen and Fluorine. Phys. Rev. B 81, 085442 (2010).

14. Topsakal, M., Aktürk, E. \& Ciraci, S. First-principles Study of Two- and Onedimensional Honeycomb Structures of Boron Nitride. Phys. Rev. B 79, 115442 (2009).

15. Heyd, J., Scuseria, G. E. \& Ernzerhof, M. Hybrid Functionals Based on a Screened Coulomb Potential. J. Chem. Phys. 118, 8207-8215 (2003).

16. Heyd, J., Scuseria, G. E. \& Ernzerhof, M. Erratum: "Hybrid functionals based on a screened Coulomb potential” [J. Chem. Phys. 118, 8207 (2003)]. J. Chem. Phys. 124, 219906 (2006)

17. Paier, J., Marsman, M., Hummer, K. \& Kresse, G. Screened Hybrid Density Functionals Applied to Solids. J. Chem. Phys. 124, 154709 (2006).

18. Marsman, M., Paier, J., Stroppa, A. \& Kresse, G. Hybrid Functionals Applied to Extended Systems. J. Phys.: Condens. Matter 20, 064201 (2008).

19. Song, L. et al. Large Scale Growth and Characterization of Atomic Hexagonal Boron Nitride Layers. Nano Lett. 10, 3209-3215 (2010).

20. Weast, R. C., Astle, M. J. \& Beyer, W. H. Handbook of Physics and Chemistry (64th edn, CRC Press, 1983).

21. Novoselov, K. S. et al. Electric Field Effect in Atomically Thin Carbon Films. Science 306, 666-669 (2004).

22. Sahin, H. et al. Monolayer Honeycomb Structures of Group-IV Elements and IIIV Binary Compounds: First-Principles Calculations. Phys. Rev. B 80, 155453 (2009).

23. Han, W. Q., Wu, L., Zhu, Y., Watanabe, K. \& Taniguchi, T. Structure of Chemically Derived Mono- and Few-atomic-layer Boron Nitride Sheets. Appl. Phys. Lett. 93, 223103 (2008).

24. Vogt, P. et al. Silicene: Compelling Experimental Evidence for Graphenelike TwoDimensional Silicon. Phys. Rev. Lett. 108, 155501 (2012).

25. Nair, R. et al. Fluorographene: A Two-Dimensional Counterpart of Teflon. Small 6, 2877-2884 (2010).

26. Bhattacharya, A., Bhattacharya, S. \& Das, G. P. Band Gap Engineering by Functionalization of BN Sheet. Phys. Rev. B 85, 035415 (2012).

27. Ding, Y. \& Wang, Y. Electronic Structures of Silicene Fluoride and Hydride. Appl. Phys. Lett. 100, 083102 (2012).

28. Garcia, J., Lima, D., Assali, L. \& Justo, J. Group IV Graphene- and Graphane-Like Nanosheets. J. Phys. Chem. C 115, 13242-13246 (2011).

29. Perdew, J. P., Burke, K. \& Ernzerhof, M. Generalized Gradient Approximation Made Simple. Phys. Rev. Lett. 77, 3865-3868 (1996). 
30. Kresse, G. \& Furthmüller, J. Efficient Iterative Schemes for ab Initio Total-Energy Calculations using a Plane-Wave Basis Set. Phys. Rev. B 54, 11169-11186 (1996).

31. Blöchl, P. E. Projector Augmented-Wave Method. Phys. Rev. B 50, 17953-17979 (1994).

32. Kan, E. J. et al. Half-Metallicity in Organic Single Porous Sheets. J. Am. Chem. Soc. 134, 5718-5721 (2012)

33. Gajdoš, M., Hummer, K., Kresse, G., Furthmüller, J. \& Bechstedt, F. Linear optical properties in the PAW methodology. Phys. Rev. B 73, 045112 (2006).

\section{Acknowledgments}

This work is partially supported by the National Key Basic Research Program (2011CB921404), by NSFC $(21121003,21233007,91021004,21003113)$, by CAS (XDB01020300), and by USTCSCC, SCCAS, Tianjin, and Shanghai Supercomputer Centers.

\section{Author contributions}

J.Y. conceived the idea. X.L. carried out the first-principles calculations. X.L., J.Z. and J.Y. analyzed the results and wrote the paper.

\section{Additional information}

Competing financial interests: The authors declare no competing financial interests.

License: This work is licensed under a Creative Commons

Attribution-NonCommercial-NoDerivs 3.0 Unported License. To view a copy of this license, visit http://creativecommons.org/licenses/by-nc-nd/3.0/

How to cite this article: Li, X., Zhao, J. \& Yang, J. Semihydrogenated BN Sheet: A Promising Visible-light Driven Photocatalyst for Water Splitting. Sci. Rep. 3, 1858; DOI:10.1038/ srep01858 (2013) 